\title{
Using the EDS Clues: Peak Fitting Residual Spectrum and Analytical Total
}

\author{
Dale E. Newbury and Nicholas W. M. Ritchie \\ National Institute of Standards and Technology, Gaithersburg, MD 20899-8370
}

Electron-excited X-ray microanalysis with energy dispersive X-ray spectrometry (EDS) can provide comprehensive elemental analysis for all elements, except for $\mathrm{H}$ and $\mathrm{He}$ which do not produce X-rays. However, the poorer spectral resolution, $\sim 125 \mathrm{eV}$ full peak width at half peak maximum (FWHM) as compared to $\sim 8 \mathrm{eV}$ for wavelength dispersive spectrometry (WDS) at Mn K- $\mathrm{L}_{2,3}(5890 \mathrm{eV})$, leads to frequent peak interference situations in EDS spectra. When interferences occur between the available analytical characteristic X-ray peaks, it is possible that one of the constituents will not be recognized during qualitative analysis (X-ray peak assignment), even for interferences of major-upon-major constituents (where "major" is arbitrarily defined as a mass concentration $C_{i}>0.1$; "minor" is $0.01 \leq C_{i} \leq 0.1$, and trace is $C_{i}<0.01$ ). For interference involving major-upon-minor or major-upon-trace constituents, it is much more likely that the minor or trace constituent will not be recognized. To detect such hidden peaks, the EDS analytical software should construct the "residual spectrum" that remains after peak fitting of the complete X-ray families of the known constituents to extract characteristic X-ray intensities necessary for the subsequent quantitative analysis. Moreover, if this quantitative analysis is performed by following the standards-based k-ratio protocol with matrix corrections (for electron scattering, X-ray absorption, and secondary X-ray fluorescence) originated by Castaing, then the raw analytical total, i.e., the sum of all constituents including any element such as oxygen calculated by the method of assumed stoichiometry, can also suggest the possibility of missing constituents through deviation below unity [1,2]. The following example illustrates the use of these tools for the analysis of NIST SRM $481 \mathrm{Au}-\mathrm{Ag}$ alloys (nominal 20Au-80Ag). The surface being analyzed was metallographically prepared in the early 1970s and stored in a desiccator. In Figure 1 (upper), the Au M-family and Ag L-family appear to account for all the X-ray peaks in this energy range. Quantitative analysis with pure element standards and NIST DTSA-II reveals anomalously low analytical totals with the $\mathrm{Ag} \mathrm{L}$-family and either the $\mathrm{Au} \mathrm{L}_{3}-\mathrm{M}_{4,5}(0.9040)$ or the Au M-family (0.8382). Note that if the normalized concentrations are used, the relative deviation from expected value (RDEV) calculated as:

RDEV $=[($ Measured-True $) /$ True $] \times 100 \%$

is modest for analysis with $\mathrm{Ag} \mathrm{L}_{3}-\mathrm{M}_{4,5}(-0.79 \%)$ and $\mathrm{Au} \mathrm{L}_{3}-\mathrm{M}_{4,5}(2.7 \%)$, although the result for Au has a large RDEV value for analysis with $\mathrm{Ag} \mathrm{L}_{3}-\mathrm{M}_{4,5}$ (3.4\%) and the Au M-family (-11.8\%). Close inspection of the peak fitting residual spectrum shown in Figure 1 (lower) reveals the reason for the low totals: $\mathrm{S}$ and $\mathrm{Cl}$ are present within a surface tarnish layer, but the S K-family is hidden under the Au M-family, while the $\mathrm{Cl} \mathrm{K}$-family is hidden under the Ag L-family. Including these elements in the analysis reveals S (0.0162) and $\mathrm{Cl}(0.0105)$ and increases the $\mathrm{Ag}$ and $\mathrm{Au}$ concentrations due to improved fitting, raising the analytical total to 0.9945 for analysis with $\mathrm{Ag} \mathrm{L}$-family and $\mathrm{Au} \mathrm{L}_{3}-\mathrm{M}_{4,5}$, and to 0.9730 for analysis with the Au Mfamily. When analyzed at low beam energy $(5 \mathrm{keV})$, the tarnish layer comprises a larger fraction of the sampling depth, and the $\mathrm{S}$ and $\mathrm{Cl} \mathrm{K}$-family peaks become much more obvious, as shown in Figure 2 . Quantitative analysis at $\mathrm{E}_{0}=5 \mathrm{keV}$ yields an analytical total of 0.9961 and an $\mathrm{Ag} / \mathrm{Au}$ ratio of $8.3 \mathrm{compared}$ to 3.5 expected from the SRM values. Much higher levels of S (0.0949) and $\mathrm{Cl}(0.0905)$ are found. The $\mathrm{Ag}$ content of the surface layer is likely enriched relative to Au due to the formation of $\mathrm{Ag}$ sulfide and chloride in the tarnish layer. Trace $\mathrm{Cu}(0.0082)$, likely deposited on the surface during the original polishing by transfer from a nearby co-mounted $\mathrm{Cu}$ standard, is also detected in the tarnish layer.

1. Goldstein J.I., Newbury, D.E., Michael, J.R., Ritchie, N.W.M., Scott, J.H.J., and Joy, D.C., Scanning Electron Microscopy and X-ray Microanalysis, $4^{\text {th }}$ ed. (Springer, New York, 2018).

2. Castaing, R., Ph. D. Thesis, "Application of electron probes to local chemical and crystallographic analysis," University of Paris (1951).

3. Ritchie, N.W.M. (2018). NIST DTSA-II software, including tutorials. Available for free at: www.cstl.nist.gov/div837/837.02/epq/dtsa2/index.html (retrieved November 27, 2018). 

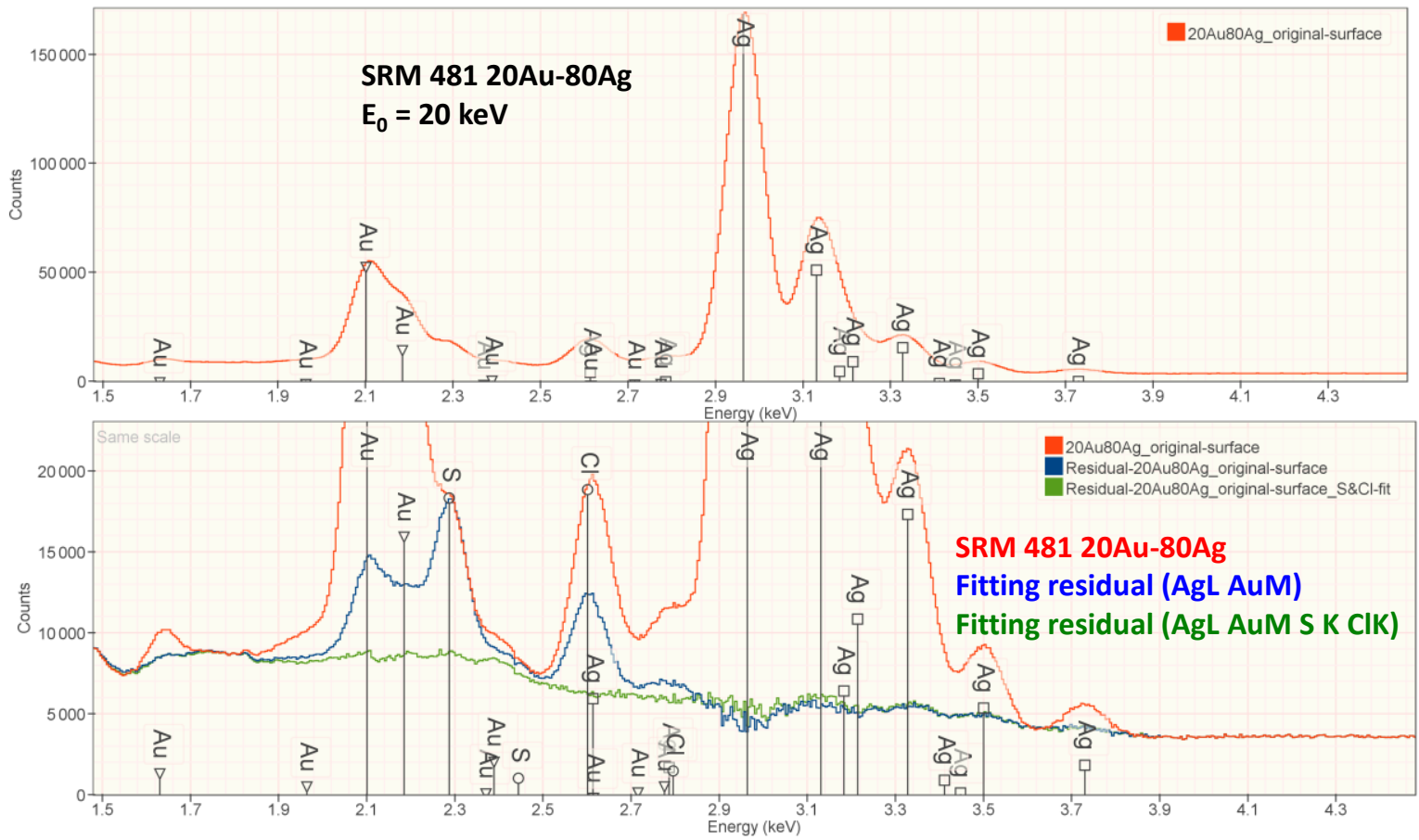

Figure 1. NIST SRM 481 (20Au-80Ag alloy) $\left(\mathrm{E}_{0}=20 \mathrm{keV}\right)$ spectrum (1970s metallographic preparation) (upper, red trace) and the peak fitting residual spectrum (lower) after fitting for the AgL and AuM families only (blue trace) and after including the $\mathrm{S}$ and $\mathrm{Cl} \mathrm{K}$-families (green trace) in the fitting suite.

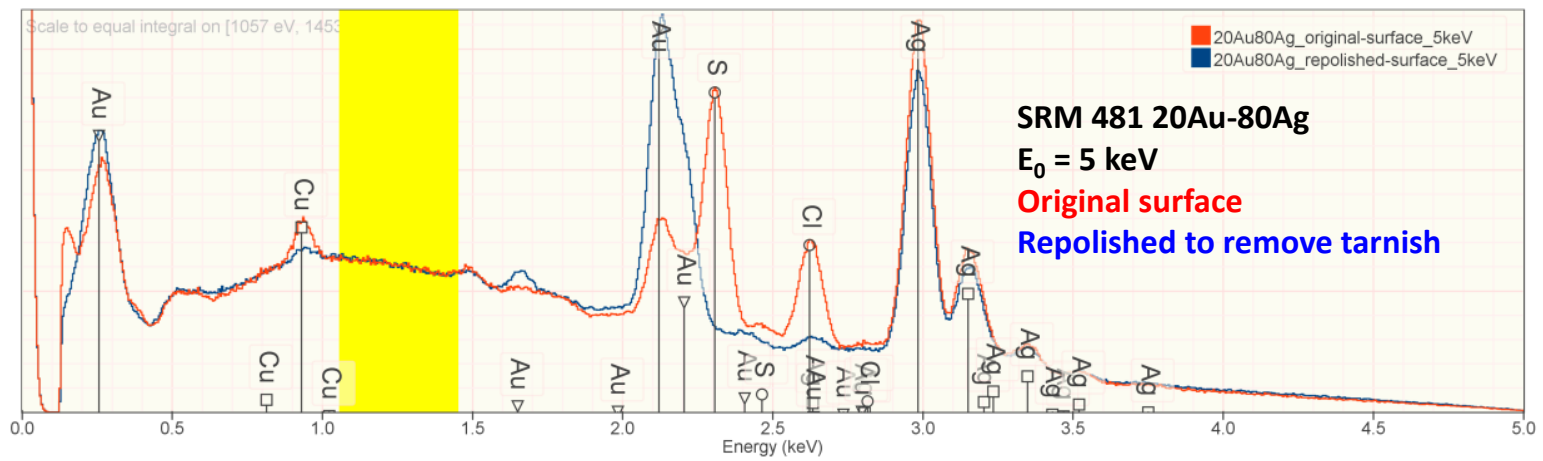

Figure 2. NIST SRM 481 (20Au-80Ag alloy) $\left(\mathrm{E}_{0}=5 \mathrm{keV}\right) 1970$ s preparation (red trace) and after polishing to remove surface tarnish layer (blue trace). Spectra scaled to highlighted region. 